\title{
A sobrecarga de cuidadores de pacientes com esquizofrenia
}

\author{
The burden in caregivers of schizophrenic patients
}

\author{
Marcelo Machado de Almeida ${ }^{1}$, Virgínia Torres Schal ${ }^{2}$, Alberto Mesaque Martins ${ }^{3}$, Celina Maria Modena ${ }^{4}$ \\ ${ }^{1}$ Mestre em Ciências da Saúde, Centro de Pesquisa René Rachou (CPqRR), Fundação Oswaldo Cruz (Fiocruz). Médico psiquiatra, Centro de Atenção Psicossocial \\ (CAPS), Lagoa da Prata, MG. ${ }^{2}$ Doutora em Educação. Psicóloga. Pesquisadora titular, Conselho Nacional de Desenvolvimento Científico e Tecnológico/Fundação \\ de Amparo à Pesquisa do Estado de Minas Gerais (CNPq/FAPEMIG) e Laboratório de Educação em Saúde e Ambiente (LAESA), CPqRR, Fiocruz. ${ }^{3}$ Acadêmico de \\ Psicologia, Centro Universitário UNA, Belo Horizonte, MG. Bolsista de Iniciação Científica, CNPq, LAESA, CPqRR, Fiocruz. ${ }^{4}$ Pós-Doutora em Saúde Coletiva. \\ Psicóloga. Pesquisadora visitante CNPq/FAPEMIG, LAESA, CPqRR, Fiocruz.
}

\section{Resumo}

Introdução: A reforma psiquiátrica brasileira conduziu a novas práticas de assistência à saúde mental, associadas ao processo de desospitalização. Deixando os hospitais, os pacientes portadores de esquizofrenia passaram a ser tratados dentro da comunidade, e um novo sujeito passou a ganhar força nessa modalidade de tratamento: o cuidador.

Objetivo: Avaliar os fatores de sobrecarga em cuidadores de pacientes esquizofrênicos.

Método: Os fatores de sobrecarga foram avaliados utilizando metodologia qualitativa, apropriada para analisar o mundo dos significados, motivos, crenças, aspirações, valores e atitudes dos cuidadores. O estudo foi realizado num Centro de Atenção Psicossocial do interior de Minas Gerais. Foram entrevistados um total de 15 cuidadores, selecionados com base nos critérios de saturação e singularidade das falas. As entrevistas foram analisadas na perspectiva do método fenomenológico.

Resultados: Nos discursos dos cuidadores é possível identificar que as atividades da vida diária, as mudanças na rotina, a diminuição do lazer, os problemas de saúde, as preocupações, o medo de adoecer, a obrigatoriedade do cuidado, o custo do tratamento, aspectos financeiros e expectativas em relação ao futuro são importantes fatores de sobrecarga. Foram identificados quatro fatores de redução da sobrecarga: apoio social e família, presença de espaços para aliviar as tensões, afastamento físico do paciente e valorização do tratamento farmacológico.

Conclusão: Fazem-se necessários programas de atendimento específicos para essa parcela da população, já que o discurso dos cuidadores apontou que o cuidado de pacientes com esquizofrenia pode impactar negativa e permanentemente as suas vidas.

Descritores: Cuidadores, esquizofrenia, família, pesquisa qualitativa.

\begin{abstract}
Introduction: The Brazilian psychiatric reform has led to new mental health care practices associated with the de-hospitalization process. Previously treated at hospitals, patients suffering from schizophrenia now receive treatment within the community, shedding light on a new type of care provider involved in this treatment modality, namely the caregiver.

Objective: To assess factors associated with burden in caregivers of schizophrenic patients.

Method: Burden factors were assessed using a qualitative method appropriate to the analysis of meanings, motivations, beliefs, aspirations, values, and attitudes. The study was conducted at a Psychosocial Attention Center located in the countryside of the state of Minas Gerais, Brazil. A total of 15 caregivers were interviewed, selected based on the criteria of saturation and singularity of speeches. The phenomenological method was used to analyze the interviews.

Results: The speeches of caregivers revealed the following factors as significantly associated with burden: daily care activities, changes in the routine, decrease in leisure, health problems, preoccupations, fear of becoming sick, obligation of providing care, treatment costs, financial aspects, and expectations for the future. Four factors associated with the reduction of burden were identified: social and family support, availability of places/opportunities for caregivers to relieve tensions, physical distance from the patient, and appreciation of the pharmacological treatment.

Conclusion: There is a need to implement programs designed specifically for caregivers, once their speeches have suggested that the care of schizophrenic patients may have a negative and permanent impact on the lives of these care providers.
\end{abstract}

Keywords: Caregiver, schizophrenia, family, qualitative research.

\section{Correspondência:}

Celina Maria Modena, Centro de Pesquisas René Rachou, Fundação Oswaldo Cruz MG, Av. Augusto de Lima, 1715, sala 102, CEP 30190-002, Barro Preto, Belo Horizonte, MG. E-mail: celina@cpqrr.fiocruz.br

Não foram declarados conflitos de interesse associados à publicação deste artigo.

Copyright (C) Revista de Psiquiatria do Rio Grande do Sul - APRS

Recebido em 19/01/2009. Aceito em 07/04/2010. 


\section{Introdução}

Com a mudança das políticas públicas em saúde mental ocorrida no Brasil no século XX, prevendo a desospitalização dos enfermos, a desinstitucionalização psiquiátrica e a consequente ênfase em serviços comunitários de saúde mental, a família dos pacientes passou a ganhar um lugar privilegiado no que tange às estratégias de tratamento e cuidado ${ }^{1}$. Todavia, as famílias foram inseridas no processo da reforma psiquiátrica sem a preparação e o conhecimentos necessários para desempenhar seu papel.

Na maioria das vezes, a pessoa que realiza a função de cuidador não está preparada para exercê-la ${ }^{2}$. Os cuidados podem incluir, além de auxílio em atividades da vida diária ou da vida prática, o acompanhamento do tratamento como um todo. Nesse sentido, o cuidador pode tornar-se um importante aliado na atenção, dependendo da forma como ele concebe o tratamento e as questões relacionadas à saúde, bem como de sua relação com os serviços e os profissionais ${ }^{3}$.

Sobrecarga familiar (family burden) é o impacto causado no meio familiar pela convivência com o paciente, envolvendo aspectos econômicos, práticos e emocionais aos quais os cuidadores/familiares são submetidos ${ }^{4,5}$. Familiares sobrecarregados têm uma demanda de apoio por parte de profissionais de saúde no sentido de obter maior suporte social e mais informações sobre os transtornos mentais e sobre como lidar com os pacientes durante a crise $\mathrm{e}^{6,7}$.

Vários fatores podem influenciar a sobrecarga dos cuidadores, tais como características dos pacientes, grau de parentesco, frequência do convívio entre cuidadores e pacientes, a personalidade dos cuidadores, suporte social, estrutura e adequação dos serviços de saúde ${ }^{8,9}$.

Estudos brasileiros e internacionais demonstraram que cuidadores de pacientes portadores de condições crônicas e incapacitantes experienciam algum tipo de sobrecarga, seja ela objetiva ou subjetiva ${ }^{10-12}$. Garrido \& Menezes ${ }^{13}$, avaliando o impacto das atividades de cuidado em cuidadores de idosos com síndrome demencial, observaram altas taxas de impacto sobre a vida social, sobre o bem-estar físico e emocional e sobre as finanças dos cuidadores. Os autores apontam para a necessidade de implementar ações no sentido de apoiar as famílias dos enfermos, evitando, assim, danos tanto para os cuidadores como para os pacientes.

Com o objetivo de identificar estudos sobre cuidado em famílias de portadores de transtorno mental no paradigma da desinstitucionalização, Waidman ${ }^{2}$ destacou que estudos nessa área são escassos no Brasil e raramente abordam a questão da sobrecarga, mas apontam para a necessidade do aprimoramento do cuidado com o cuidador e para uma mudança de paradigma na atenção oferecida pelos profissionais de saúde, no intuito de valorizar a reinserção social e o resgate da cidadania do paciente.

Barroso et al. ${ }^{8}$, avaliando a sobrecarga de cuidadores de pacientes psiquiátricos atendidos na rede pública, identificaram que $84 \%$ dos pacientes residiam com seus familiares.
Os autores associam essa variável a um maior nível de sobrecarga, já que o convívio com o paciente na mesma residência pode contribuir para a elevada frequência de tarefas de assistência realizadas pelos cuidadores no cotidiano. A associação entre convivência e sobrecarga foi demonstrada em estudos internacionais, como o de Provencher et al. ${ }^{14}$, que encontrou uma significativa associação entre convivência com o paciente e estresse em cuidadores de pacientes psiquiátricos canadenses.

A importância do cuidador, a escassez de estudos sobre o tema e a necessidade de estudos contextualizados em diferentes cenários socioeconômicos reitera a necessidade de pesquisas sobre o assunto. Assim, este estudo teve como objetivo identificar fatores de sobrecarga vivenciados por cuidadores de pacientes portadores de esquizofrenia atendidos em um serviço público de saúde mental de um município do interior de Minas Gerais.

\section{Metodologia}

\section{Referencial teórico}

Elegeu-se a metodologia qualitativa por ser a mais adequada para conduzir uma investigação aprofundada do mundo de significados, motivos, crenças, aspirações, valores e atitudes dos cuidadores ${ }^{15}$. O referencial teórico que balizou o estudo foi o da fenomenologia, que interroga a experiência vivida e busca captar o significado atribuído à relação sujeito-objeto - neste caso específico, a relação cuidador/paciente. A fenomenologia, redefinida por Heidegger, tem como um dos seus pressupostos a máxima hursseliana de "ir às coisas mesmas", isto é, buscar como o sujeito vê, interpreta e vive determinada situação, qual o enfoque que ele dá às coisas, como ele apreende a realidade com as diferentes formas de viver e como estabelece relações com os outros entes na construção da intersubjetividade ${ }^{16,17}$.

Optou-se pela fenomenologia porque ela permite abordar a existência numa perspectiva experiencial, ou seja, o universo do homem é composto pelo seu contexto sócio-histórico e pela linguagem ${ }^{18}$, e cada cuidador vivencia o cuidado de maneira singular.

\section{Descrição do local e dos participantes}

O estudo foi realizado no município de Lagoa da Prata (MG), que tem uma população média de 44.000 habitantes $^{19}$. Há apenas um serviço público de saúde mental para adultos disponível no município, que atende cerca de 600 pacientes/ mês e seus respectivos cuidadores.

Os pares de cuidadores e pacientes foram identificados a partir dos prontuários arquivados no Centro de Atenção Psicossocial (CAPS). Foram selecionados cuidadores de pacientes com diagnóstico de esquizofrenia segundo os critérios da Classificação Internacional das Doenças, $10^{\mathrm{a}}$ edição 
$(\mathrm{CID}-10)^{20}$. Os critérios de seleção utilizados foram: cuidar de um paciente com esquizofrenia, residir no município de Lagoa da Prata, concordar com a participação no estudo e assinar o termo de consentimento livre e esclarecido.

Uma vez identificados os pares que atendiam aos critérios pré-estabelecidos, foi feito um primeiro contato, por parte de um dos pesquisadores, para explicar os objetivos da pesquisa via telefone ou pessoalmente. Em seguida, foi agendada uma entrevista realizada no horário e local de maior conveniência para o cuidador (residência ou serviço de saúde).

O número de participantes foi determinado a partir dos critérios de saturação e singularidade do discurso, segundo pressupostos descritos por Minayo ${ }^{15}$. De acordo com essa perspectiva teórica e metodológica, o número adequado de entrevistas deve ser entendido como aquele capaz de refletir a totalidade nas suas dimensões. Nesse sentido, há uma suspensão da inclusão de novos participantes quando os dados obtidos passam a apresentar, na avaliação do pesquisador, uma certa redundância ou repetição ${ }^{21}$.

Todos os participantes concordaram em participar da pesquisa e, em seguida, assinaram o termo de consentimento livre e esclarecido. O estudo foi aprovado pelo Comitê de Ética em Pesquisa com Seres Humanos do Centro de Pesquisa René Rachou, Fundação Oswaldo Cruz MG (parecer no. 02-2008, protocolo no. 01-2008), em concordância com a Resolução no 196/96 do Conselho Nacional de Saúde.

\section{Procedimentos}

Foram realizadas entrevistas semiestruturadas com os cuidadores, tendo como eixos temáticos temas relacionados à sobrecarga, ou seja, cotidiano, vivências e experiências na relação do cuidado. A entrevista foi construída considerando as características sociodemográficas da população atendida no CAPS.

As entrevistas duraram em média 30 minutos, foram gravadas e transcritas literalmente e na íntegra, e depois analisadas de acordo com as etapas do método fenomenológico, que incluem descrição, redução e compreensão. A descrição objetiva captar a essência do fenômeno, buscando as unidades de significado. Na redução, determinam-se e selecionamse as partes essenciais da descrição. Durante a compreensão, faz-se um movimento contínuo do individual para o geral, que revela as convergências, divergências e idiossincrasias de cada caso em particular 22,23 .

O discurso dos cuidadores foi analisado quanto à caracterização da sobrecarga segundo os critérios utilizados por Barroso et al. ${ }^{8}$ e Grandón et al. ${ }^{24}$, que diferenciam a sobrecarga em duas dimensões: objetiva e subjetiva. A análise das entrevistas permitiu a identificação da categoria redução da sobrecarga.

\section{Resultados e discussão}

\section{Caracterização dos cuidadores}

Foram entrevistados 15 cuidadores de pacientes que atendiam aos critérios pré-estabelecidos. Deve-se considerar que nenhum dos pacientes/cuidadores convidados se recusou a participar da pesquisa, portanto todas as entrevistas foram analisadas.

Em relação às características sociodemográficas dos cuidadores, nove eram mulheres e seis eram homens, com renda familiar variando de um a cinco salários mínimos. A idade média dos cuidadores era de 48 anos (25-84). Quanto ao vínculo familiar com o paciente, cinco eram irmãos, quatro eram genitores, dois eram cônjuges, dois eram filhos, um era tio e um não era familiar. Predominou a escolaridade caracterizada como ensino fundamental incompleto. A maior parte dos pacientes era do sexo feminino (8), com idade média de 41 anos (25-65).

\section{Sobrecarga objetiva}

A sobrecarga objetiva se refere às consequências da alteração da rotina e dos projetos de vida, da diminuição da vida social, da supervisão de comportamentos problemáti$\cos$, dentre outros ${ }^{25}$. O cuidado com o paciente portador de um transtorno mental grave requer adaptações na vida diária, e os cuidadores acabam por desistir ou mudar os seus objetivos, deixando de viver sua realidade e passando a viver uma realidade construída a partir do convívio com o paciente ${ }^{26}$. As falas a seguir traduzem este modo de viver:

Às vezes eu quero ir à missa, não vou (...) eu fui à missa aquela quarta-feira de cinzas (...) dentro da igreja ela me deu uma cabeçada aqui, fez um galo (...) chega lá ela vai fazer confusão pra mim. (Papoula*)

Em alguns casos, a necessidade de mudança da rotina traz consequências para os planos futuros:

Preciso casar e ela não aceita. E eu também fico com dó de casar e deixar ela sozinha com meu pai, que eu cuido mais dela que meu pai cuida. (Orquídea)

Uma das primeiras coisas de que o cuidador abre mão é o lazer. A restrição ao lazer pode ter implicações na qualidade de vida dos cuidadores, sendo um dos parâmetros para a avaliação da sobrecarga ${ }^{27}$. A falta de lazer como limitante de uma vida social apareceu em várias falas, como exemplificado nas que se seguem:

Ah, mudou muita coisa. (...) pra sair com ela é dificil, (...) como é que sai e deixa ela em casa, sai ela dá problema. Muito dificil. (Girassol)

* Todos os nomes que aparecem no texto são fictícios. 
Eu vou pra casa da minha mãe, ai eu levo ele (...) e lá fico cuidando dele também. (Jasmim)

Sob um prisma mais específico, mesmo sem representar uma alteração propriamente dita da rotina, as questões de ordem prática, como os cuidados na higiene, alimentação, entre outros, podem representar uma sobrecarga efetiva na medida em que demandam supervisão e auxílio na realização das atividades, que são diárias.

É só eu, tudo é eu, até uma colher pra ela comer eu tenho que lavar, é só eu tudo, nem uma pecinha assim ela não lava. (Papoula)

$\dot{E}$, num sabe fazer uma comida pra ele (...) se deixar ele sozinho uma semana numa casa, na hora que ocê chegar perigoso ocê encontrar ele ou morto ou de ter um bicho lá dentro (...) porque ele não tem mesmo noção de limpeza, de afazeres, essas coisas, então minha mãe na idade que ela tá (...) não tem condições de cuidar dele mais. (Gerânio)

A sobrecarga de ordem prática, traduzida pela dependência do paciente, gera uma tensão cotidiana no cuidador, que agrega essas atividades a outras responsabilidades da vida diária. Esse aspecto se agrava ainda mais em cuidadores que sobrevivem com baixa renda ${ }^{12,28}$.

A saúde é um dos parâmetros mais importantes a ser contemplado quando se considera a qualidade de vida de um indivíduo. Um estudo conduzido para avaliar a qualidade de vida de familiares cuidadores de esquizofrênicos demonstrou que a saúde vem sempre em primeiro lugar na concepção de qualidade de vida relatada pelos participantes ${ }^{29}$. No presente estudo, foram observados relatos de problemas de saúde diretamente associados ao cuidado com o paciente, à convivência, aos hábitos dos pacientes ou mesmo a agressões:

Que pra mim é demais meu filho (...) ai eu não aguento, que ela me puxa pra cama, da cama puxa o cabelo (...) vou guentando, passo a noite sem dormir, ai perco a vontade de comer (...) esse ano já fui umas quatro vezes pro balão (de oxigênio) (...) num tem vida, né (...) o cigarro dela me colocou doente. (Papoula)

Igual eu te falei que eu tenho problema de pressão, minha pressão vive alta, eu vivo no pronto-socorro direto (...). Já fiz tratamento com psicólogo. Eu fiquei doente demais e na época eu perdi $15 \mathrm{~kg}$. (Alfazema)

A dimensão financeira assumiu uma importância expressiva no discurso dos cuidadores: as falas apontaram para o custo do tratamento, a diminuição da produtividade dos pacientes e a interferência na atividade profissional dos cuidadores. Considerando que, no contexto estudado, a renda familiar era de até cinco salários mínimos, observou-se que o custo do tratamento exerce um peso significativo no orçamento doméstico, conforme expressaram os cuidadores:

O mês (...) que não tem medicamento aqui, tem que comprar (...) às vezes salário nem dá pro medicamento, que ele toma nove remédio por dia. (Crisântemo)

Eu trabalho de doméstica, eu também ganho muito pouco (...). E ela gasta muito, às vezes a medicação dela é cara, tem que tá pagando condução pra trazer ela aqui. (Orquídea)

Em função da irregularidade no fornecimento de medicação e da dificuldade com o transporte, os custos com a esquizofrenia muitas vezes recaem sobre os cuidadores. Existem recursos públicos disponíveis para cobrir essas despesas, como garante o Sistema Único de Saúde (SUS), mas um estudo realizado em São Paulo apontou para a necessidade de uma melhor alocação dos recursos a fim de cobrir os custos com a esquizofrenia ${ }^{\dagger 30}$.

Outra questão difícil de ser administrada é a diminuição de produtividade que a esquizofrenia frequentemente traz. Considerando que a renda familiar é menor entre os usuários do sistema público de saúde, o sustento do paciente pouco produtivo pesa no orçamento. Muitos pacientes nunca tiveram rendimentos; já outros, que por vezes exerciam a função de provedores ou até de "arrimo", saem dessa posição para se tornarem dependentes. Ao familiar cabe o ônus de redimensionar os gastos.

Teve um impacto no início, teve porque ele tava trabalhando (...). Começou a dá loucuras lá no serviço e foi mandado embora e não tinha dinheiro. (Gerânio)

Era normal, trabalhava, tudo direitinho. Sempre foi o nosso arrimo. Trabalhava, (...) se ele trabalhasse (...) tenho certeza que minha irmã ia achar muito melhor que aí já ajudava um pouquinho. (Cravo)

Um estudo qualitativo, que teve como objetivo analisar as consequências da reforma psiquiátrica brasileira sobre o cuidado do doente mental na família, já demonstrava que os cuidadores expressam sofrimento, tensões e conflitos diante da sobrecarga financeira que a diminuição da produtividade de um membro da família acarreta ${ }^{32}$.

Por vezes, a tarefa de cuidar exige rearranjos na situação laborativa dos cuidadores. A proximidade que o cuidado exige pode acarretar mudanças na rotina de trabalho ou mesmo a decisão de deixar o emprego a despeito da perda financeira que isso possa causar:

Agora eu (o pai) tive que largar o trabalho que eu tava fazendo pra ficar com ele; que tava ficando perigoso ela (a mãe) ficar sozinha, eu ficava até quarenta dias fora de casa, eu viajo de motorista. (Lírio)

† O SUS é um dos maiores sistemas públicos de saúde do mundo, criado em 1988 pela Constituição Federal Brasileira. Ancorado nos princípios da universalidade, integralidade e equidade, o SUS abrange desde o simples atendimento ambulatorial até o transplante de órgãos, garantindo acesso integral, universal e gratuito para toda a população do país ${ }^{31}$. 
Eu já saí do serviço umas três vezes (...) tive que sair pra cuidar dela. (Orquídea)

\section{Sobrecarga subjetiva}

A sobrecarga subjetiva se refere a distúrbios emocionais experienciados pelo cuidador ou às percepções, preocupações, sentimentos negativos e incômodos gerados pelo fato de ter que cuidar de um paciente esquizofrênico ${ }^{33}$.

De maneira geral, os entrevistados afirmaram que o cuidar assume um caráter de obrigatoriedade, como se não houvesse a possibilidade de escolha, ou seja, cuidam porque têm que cuidar, como se fosse um destino imutável e inexorável:

Não, o cuidado dela é meu, né, que ela é minha companheira, eu sou casado com ela, eu tenho que zelar o que eu der conta. (Alecrim)

Para esse tipo de cuidador, não se admite a hipótese de delegar a outro o cuidado de alguém por quem se nutra um afeto próximo, como no caso de cônjuges e filhos. Esse fato também foi demonstrado de maneira enfática em estudo internacional ${ }^{34}$. Outra forma de sentir-se impelido a cuidar, observada no presente estudo, é aquela em que um outro significativo para o cuidador outorga-lhe a missão do cuidado:

A minha familia me julgou assim de mão beijada, porque eles acham que eu que tenho que cuidar. Então é por isso e a gente ficou tudo de mal (...) e eu que cuido dele sozinha por causa disso. (Alfazema)

$E$ antes da minha mãe morrer mais o meu pai, eles falou: Hortênsia, se algum dia cê casar, cê faz um barracão no fundo, num larga a Camélia mais Azaléia não. Não deixa elas de mão; antes dela falecer sabe. Que aí eu não largo elas, eu tomo conta delas. (Hortênsia)

Nessas falas, podemos nos aproximar do conceito de facticidade proposto por Heidegger, que consiste no fato de o "ser" estar no mundo à mercê de situações sem necessariamente participar das decisões relacionadas: simplesmente surge uma contingência, e o sujeito tem que aprender a lidar com ela ${ }^{17}$.

Outro fator importante a ser considerado são as preocupações. Muitos cuidadores sentem-se como se nunca estivessem totalmente tranquilos, pois as preocupações os acompanham dia a dia, principalmente na supervisão de comportamentos que o paciente adota ou pode adotar, como tentativas de fuga, suicídio e realização de atividades diárias.

Eu tenho medo igual eu te falei, muito medo dele suicidar (...) tem um tanto de paciente lá, você num sabe a hora da reação dele, você num vai sabê, ele pode enforcar qualquer hora porque ele não acha a vida boa. Num acha de jeito nenhum. (Alfazema)

Às vezes eu tô trabalhando, eu fico preocupada lá em casa com ela, porque eu tenho medo dela não saber mexer no fogão, queimar. Ou vez passar mal e cair sozinha e não ter ninguém pra cuidar dela em casa. (Orquídea)

Em um estudo qualitativo realizado com familiares de um CAPS, foi observada sobrecarga em relação às fugas e às tentativas de suicídio dos pacientes ${ }^{28}$. Diante da possibilidade de suicídio, os familiares sentem-se impotentes e agem no sentido de evitar tal comportamento, sendo essa uma situação limite ${ }^{32}$. O risco de suicídio também aparece como um fator que pode gerar sensação de culpa por não ter sido percebido antes ${ }^{34}$.

Os cuidadores entrevistados no presente estudo também relataram medo de adoecer fisicamente, o que se relaciona com a sobrecarga objetiva, com o cansaço e com o medo de adoecer psiquicamente. Este último aspecto está associado com o convívio diário com o paciente e a sensação de vulnerabilidade que isso causa, como se o cuidador pudesse "ficar igual" ao paciente:

Eu passei por uma carga forte mas eu venci, graças a Deus eu venci (...) não precisei assim ser usuária do CAPS (...). (Margarida)

Às vezes pode dar um surto psicótico em mim e eu ficar igual a ela. (Orquídea)

Isso aí acho que ajudou muito na doença da mãe (que morreu de câncer). (Crisântemo)

A expectativa em relação ao futuro, explicitada na preocupação com a existência de um cuidador substituto (caso os atuais venham "a faltar"), esteve presente na maioria das falas:

Uai, eu penso assim, porque a Camélia falô que não casa mais; eu acho que a Azaléia também não; e na falta minha como é que elas vão fazer, né? (Hortênsia)

(...) na minha falta, eu tenho mais filhos, as noras não vai querer saber de cuidar (...) tenho uma filha só. Então o meu medo é esse. De quando eu faltar eu não sei qual vai ser o futuro dele. (Jasmim)

Outros estudos na área também identificaram como preocupação constante a possibilidade de não haver um cuidador substituto para os pacientes no futuro, ou de eles terem que ir para um asilo ou terem um futuro indeterminado ${ }^{29,34}$.

\section{Redução da sobrecarga}

$\mathrm{O}$ apoio social e familiar foi valorizado pelos entrevistados como uma alternativa de minimização da sobrecarga. Com o tratamento na comunidade, é possível contar com apoio social e familiar, dividindo-se a sobrecarga pela construção de novos vínculos sociais.

Geralmente a familia inteira ajuda a olhar um pouquinho (...) onde ele vai, quê que ele tá fazendo (...) os vizinho também fica meio preocupado (...) ao redor da casa é tudo colega dele (...) pelo menos já ajudou, já observou ele pra gente. (Cravo) 
Os cuidadores enfatizaram a necessidade de ter um espaço para aliviar as tensões, onde pudessem ser escutados e cuidados. Foi assinalada também a falta de um espaço de cuidado para com o cuidador:

A gente ter um espaço pra gente (...) um momento de relaxamento pra se desligar um pouquinho, principalmente a gente que lida com essa tensão todo dia (...) seria aconselhável pelo menos uma vez por semana. (Violeta)

A reunião de familiares foi citada como um lugar de acolhimento e troca de experiências:

Ali pra mim foi ser o meu lar, ali foi a minha família (...) eu ia lá falava na reunião. (Margarida)

Outro estudo, que analisou a qualidade de vida dos familiares cuidadores de esquizofrênicos, também identificou nas falas dos participantes a necessidade de um tempo para si mesmos ${ }^{29}$. O afastamento físico do paciente apareceu com bastante ênfase durante as entrevistas, como um fator de suavização da sobrecarga, como demonstrado nas falas abaixo:

Queria que ela viesse ficar aqui (CAPS) o dia inteiro em tratamento, né, pra ela divertir, passear, mas ela não aceita. Eu ficava menos preocupada no serviço, que eu sabia que aqui tem gente pra cuidar, pra dar comida na hora, remédio na hora. (Orquídea)

Eu acho assim que ela precisava dum lugar pra ficar sossegada... longe, entendeu? Igual da outra vez (...) nóis internou ela (...) foi uma paz. (Gardênia)

Ficou evidente, nas entrevistas, que os cuidadores valorizavam o tratamento no CAPS, como uma das possibilidades de diminuição da sobrecarga. $\mathrm{O}$ tratamento adequado tende a diminuir a dependência do cuidado, devido à melhora da sintomatologia ${ }^{12}$.

A única solução que a agente tá tendo é levar ele nos órgãos competentes, igual aquele, o CAPS (...) poder tá pegando os medicamentos e tá cuidando dele, eu acho que mudou muita coisa (...) um impacto assim estrondoso na nossa família (...) a gente não podia sair e deixar ele sozinho, né. (Gerânio)

Em algumas falas, também houve a valorização do tratamento medicamentoso, em detrimento de outros, como forma de diminuição da sobrecarga:
(...) ela toma direitinho que a gente mesmo que dá. Aquela agitação que ela tinha e outra coisa que eu acho que foi muito bom é que ela reclamava demais de dor de cabeça (...). (Tulipa)

Estudo realizado em serviços públicos de saúde mental sugere que o desenvolvimento de atividades recreativas e ocupacionais nesses serviços poderia ser um fator de diminuição da sobrecarga - além da tomada de medicação, pelo efeito direto que tem na melhora dos sintomas ${ }^{8}$. $\mathrm{O}$ tratamento medicamentoso reduz os sinais e sintomas percebidos pelo cuidador como relacionados à esquizofrenia, diminuindo, assim, a necessidade de atenção dada pelo cuidador ao paciente.

\section{Considerações finais}

A sobrecarga dos cuidadores de pacientes com esquizofrenia já é estudada na literatura mundial, mas os estudos no Brasil ainda são escassos. Com a reforma psiquiátrica, os cuidadores passaram a ter um envolvimento afetivo e social fundamental para o tratamento da doença e para a construção da cidadania do paciente. Entretanto, reconhece-se a dificuldade de estabelecer estratégias que possam suprir a complexidade desse novo paradigma. O cuidar é, de alguma forma, atribuído a pessoas por vezes ainda não adaptadas a essa nova situação.

Diante da importância que o cuidador tem no tratamento do paciente, deveriam ser propostas ações em saúde pública que contemplassem esses importantes atores no tratamento da esquizofrenia. Essas ações seriam voltadas principalmente para a redução dos agravos nas dimensões física, psíquica e social dos cuidadores.

Além disso, faz-se necessário um programa de atendimento específico para essa parcela da população, pois o discurso dos cuidadores revela que as mudanças advindas do cuidado podem impactar negativa e permanentemente as suas vidas. Devem ser feitas propostas no sentido de "cuidar do cuidador", para que este possa continuar exercendo sua função com um desgaste menor, melhorando tanto sua própria qualidade de vida quanto a daquele que é cuidado. As estratégias desenvolvidas poderiam promover a esses atores um aprendizado para uma vivência mais adaptada, por exemplo, através de espaços vivenciais para a contínua ressignificação do processo de cuidar. Considerando a integralidade da saúde, prevista como um dos princípios fundamentais do SUS, pode-se afirmar que cuidar do bem-estar de um paciente esquizofrênico inclui, também, zelar pelo seu cuidador. 


\section{Referências}

1. Hirdes A. A reforma psiquiátrica no Brasil: uma (re)visão. Rev C S Col. 2009;14(1):297-305.

2. Waidman MAP. O cuidado às famílias de portadores de transtornos mentais no paradigma da desinstitucionalização [tese]. Florianópolis: Universidade Federal de Santa Catarina; 2004.

3. Osigana VLM, Furegato ARF, Santos JLF. Usuários de três serviços psiquiátricos: perfil e opinião. Rev Latino-Am Enferm. 2007;15(1):70-7.

4. Melman J. Família e doença mental: repensando a relação entre profissionais de saúde e familiares. São Paulo: Escrituras; 2002.

5. Miles A. A família do doente mental. In: Miles A, editor. O doente mental na sociedade contemporânea. Rio de Janeiro: Zahar; 1982. p. 111-34.

6. ST-Onge M, Lavoie F. The experience of caregiving among mothers of adults suffering from psychotic disorders: factor associated with their psychological distress. Am J Community Psychol. 1997;25(1):73-94.

7. Rizzo DC, Schall VT. Representações sociais de cuidadores principais de pacientes com demência. Rev Psiquiatr Rio Gd Sul. 2008;30(1):39-48.

8. Barroso SM, Bandeira M, Nascimento E. Sobrecarga de familiares de pacientes atendidos na rede pública. Rev Psiquiatr Clin. 2007;34(6):270-7.

9. Barroso SM, Bandeira M, Nascimento E. Fatores preditores da sobrecarga subjetiva de familiares de pacientes psiquiátricos atendidos na rede pública de Belo Horizonte, Minas Gerais, Brasil. Cad Saude Publica. 2009;25(9):1957-68.

10. Tucker C, Barker A, Gregoire A. Living with schizophrenia: caring a person with severe mental illness. Soc Psychiatry Psychiatr Epidemiol. 1998;33:305-9.

11. Sena ELS, Gonçalves LHT. Vivências de familiares cuidadores de pessoas idosas com doença de Alzheimer: perspectiva da filosofia de Merleau-Ponty. Texto Contexto Enferm. 2008;17(2):232-40.

12. Pergoraro RF, Caldana RHL. Sobrecarga de familiares de usuários de um Centro de Atenção Psicossocial. Psicol Estudo. 2006;11(3):569-77.

13. Garrido R, Menezes PR. Impacto em cuidadores de idosos com demência atendidos em um serviço psicogeriátrico. Rev Saude Publica. 2004;38(6):835-41.

14. Provencher HL, Perreault M, St-Onge M, Rousseau M. Predictors of psychological distress in family caregivers of persons with psychiatric disabilities. J Psychiatr Ment Health Nurs. 2003;10:529-607.

15. Minayo MCS. O desafio do conhecimento: pesquisa qualitativa em saúde. São Paulo: Hucitec; 2007.

16. Heidegger $M$. Todos nós... ninguém: um enfoque fenomenológico do social. São Paulo: Morales; 1981.

17. Heidegger M. O ser e o tempo. Parte I. São Paulo: Vozes; 2002.
18. Garcia MLP, Jorge MSB. Vivência de trabalhadores de um Centro de Atenção Psicossocial: estudo à luz do pensamento de Martin Heidegger e Hans-Georg Gadamer. Rev C S Col. 2006;11(3):765-74.

19. Instituto Brasileiro de Geografia e Estatística. O Brasil município por município. http://www.ibge.gov.br/cidadesat/topwindow.htm?1. Acessado set 2008.

20. Organização Mundial de Saúde (OMS). Classificação de transtornos mentais e comportamentais da CID-10: descrições clínicas e diretrizes diagnósticas. Porto Alegre: Artes Médicas; 1997.

21. Fontanella BJB, Ricas J, Turato ER. Amostragem por saturação em pesquisas qualitativas em saúde: contribuições teóricas. Cad Saude Publica. 2008;24(1):17-27.

22. Dartigues A. O que é fenomenologia? São Paulo: Centauro; 2003.

23. Gomes AMA, Paiva ES, Valdés MTM, Frota MA, Albuquerque CM. Fenomenologia, humanização e promoção da saúde: uma proposta de articulação. Saude Sociedade. 2008;17(1):143-52.

24. Grandón P, Jenaro C, Lemos S. Primary caregivers of schizophrenia outpatients: burden and predictor variables. Psychiatry Res. 2008;158:335-43.

25. Provencher H, Mueser K. Positive and negative symptom behaviors and caregiver burden in the relatives of persons with schizophrenia. Schizophr Res. 1997;26:71-80.

26. Monteiro CFS, Batista DONM, Moraes EGC, Magalhães TS, Nunes BMVT, Moura MEB. Vivências maternas na realidade de ter um filho autista: uma concepção pela enfermagem. Rev Bras Enferm. 2008;61(3):330-5.

27. Scazufca M. Brazilian version of the Burden Interview Scale for the assessment of burden of care in carers of people with mental illnesses. Rev Bras Psiquiatr. 2002;24(1):12-7.

28. Pergoraro RF, Caldana RHL. Sofrimento psíquico em familiares de usuários de um Centro de Atenção Psicossocial (CAPS). Interface Comun Saude Educ. 2008;12(25):295-307.

29. Teixeira MB. Qualidade de vida de familiares de cuidadores do doente esquizofrênico. Rev Bras Enferm. 2005;58(2):171-5.

30. Leitão RJ, Ferraz MB, Chaves AC, Mari JJ. Cost of schizophrenia: direct costs and use of resources in the state of São Paulo. Rev Saude Publica. 2006;40(2):304-9.

31. Brasil, Ministério da Saúde. O que é o SUS. http://portal.saude.gov.br/portal/saude/cidadao/area.cfm?id area=1395. Acessado jan 2009.

32. Gonçalves AM, Sena RR. A reforma psiquiátrica no Brasil: contextualização e reflexos sobre o cuidado com o doente mental na família. Rev Latino-Am Enferm. 2001;9(2):48-55.

33. Schene AH, Tessler RC, Gamache GM. Instruments measuring family or caregiver burden in severe mental illness. Soc Psychiatry Psychiatr Epidemiol. 1994;29:228-40.

34. Rudge T, Morse K. Did anything change? Care givers and schizophrenia after medication changes. J Psychiatr Ment Health Nurs. 2004;11:3-11. 